\title{
Improving Starting Time in Operating Rooms of a Tertiary Hospital in Rwanda: A Quality Improvement Project
}

\author{
Assumpta Yamuragiye ${ }^{1 *}$, Faustin Ntirenganya ${ }^{1}$, David Tumusiime ${ }^{1}$, Mecthilde Mukangendo ${ }^{1}$, Dawit T Bisrat ${ }^{2}$, Rex Wong ${ }^{2}$ \\ ${ }^{1}$ College of Medicine and Health Sciences, University of Rwanda, Kigali, Rwanda \\ ${ }^{2}$ School of Medicine, Yale University, New Haven, Connecticut, United States
}

*Corresponding author: Assumpta Yamuragiye. College of Medicine and Health Sciences, University of Rwanda, Remera Campus KG 11 Ave, 47, Kigali, Rwanda. Email: assoumpta1@gmail.com

\begin{abstract}
Background

Delay in the first surgery start time at operating room (OR) could inevitably decrease utilization, lose very expensive OR resources, reduce satisfaction of patients and staff and potentially affect quality of patient care.

Objectives

This study utilized the Strategic Problem Solving (SPS) quality improvement approach to increase the percentage of first surgeries started on time at a tertiary hospital in Rwanda.

\section{Methods}

A pre- and post-intervention study was conducted between March 2016 and March 2017. The intervention included developing a policy on staff arrival time, training sessions on the policy and regular supervision of OR managers to ensure staff were arriving on time.

\section{Results}

Chi square tests were performed to analyze the pre- and post-intervention results. The percentage of first surgeries started on time significantly increased from $3 \%$ pre-intervention to $25 \%$ postintervention $(\mathrm{P}<0.001)$, average duration of delay decreased by 55 minutes $(\mathrm{P}<0.001)$ and the percentages of nurses, anesthetists and surgeon arrived on time also significantly increased $(\mathrm{P}<0.001)$.

\section{Conclusion}

The SPS approach can be useful in addressing the starting time of first surgery at OR. Support from the senior management team and buy-in from staff are essential. This project cannot eliminate confounding factors and the results cannot be generalizable to other settings. Longer term evaluation on sustainability is needed.

Rwanda J Med Health Sci 2019;2(3):289-293.
\end{abstract}

Keywords: Delay first surgery, operating room efficiency, quality improvement, strategic problem solving

\section{Introduction}

As the demand and expectations of quality health care delivery continue to increase, the hospital running cost escalates simultaneously. There is a need for hospitals to maximize the use of operating room (OR) in order to contain the ever expanding operating cost.[1] The first surgery start time has been a focus of many quality improvement initiatives in hospital OR management to maximize utilization efficiency. [1-7] When the first scheduled procedure for the day is delayed, the subsequent surgeries will be delayed and in many cases, cancelled. This inevitably will decrease the number of cases that can be performed in a single day, causing loss of very expensive OR resources and affect the satisfaction of patients and staff.[2, 8]

Improving OR efficiency has been widely studied worldwide. [1, 8] Since the operation of ORs involves a multiplicity of disciplines, many contributing factors identified have been mostly related to improper planning - including shortage of staff, lack of team work, insufficient communication and lack of planning. [3-6] In 2011, a survey conducted in India on the common causes of delay in first surgery at the OR, only one out of the top ten causes of delay was related to patients' clinical condition; the other nine were all planning and management issues. [8]

Many initiatives have been implemented to improve the first surgeries starting time, from enhancing communication among OR team members, staff education, process re-engineering.[10-13] Among all the various quality improvement approaches like Total Quality Management (TQM), Six Sigma, Lean, the eightstep Strategic Problem Solving (SPS) is a framework that emphasizes on using data and evidence to identify 
solutions to address problems in health care settings. [11] The SPS framework has been used in many health care organizations and the methodology has shown to be particularly effective in resource-limited settings. [12-16] The eight steps of SPS approach are: 1) defining the problem; 2) setting an objective; 3) conducting a root cause analysis; 4) generating alternative strategies; 5) comparing strategies; 6) selecting a strategy; 7) implementing the strategy; and 8) evaluating its impact on the problem.[17] This quality improvement project followed the SPS 8-step approach to address the delay in the starting of first surgeries in one of the referral hospitals in Kigali, Rwanda.

\section{Methods}

\section{Setting and problem description}

The study was conducted in the University Teaching Hospital of Kigali (CHUK), in Rwanda. The hospital has 560 beds and receives about $75 \%$ of all surgical referrals of the country.[18] The department of surgery has 18 surgeons, 5 anesthesiologists, 35 theater nurses, 28 nonphysician anesthesia providers and 12 supporting staff working in the six operating rooms.[19] In 2015, 6706 operations were performed; translating to approximately 3.2 surgeries per room per day.[19]

The first surgeries for the ORs were scheduled to start at 8:30AM Mondays, Wednesdays and Fridays and at 8:00AM on Tuesdays and Thursdays. Mondays, Wednesdays and Fridays have later starting times because of morning staff meetings.[19] Staff are expected to arrive at the $\mathrm{OR}$ at the following times: 7:30AM for nurse, 7:45AM for anesthetists and 8:00AM for surgeons on Mondays, Wednesdays and Fridays; and at 7:15AM for nurse, 7:30AM for anesthetists and 7:45AM for surgeons on Tuesdays and Thursdays.

A project team which included nurses, anesthesia technicians and surgeons was formed. The baseline assessment showed only $3 \%$ of first surgeries had started as scheduled. By following the SPS approach, we formed a project team to collaboratively implement the project. The team first defined the problem statement as "the percentage of first surgeries started on time was low" and had set an objective of improving the percentage of first surgeries started on time to $90 \%$. The team conducted a root cause analysis and found that many of the staff arrived at the OR later than the expected time and there was no written policy related to staff arrival time. Based on the findings, we selected and implemented an intervention to address the root cause.

\section{Design}

A pre- and post-intervention study was conducted to improve the percentage of first surgeries that started on time. The pre-intervention period baseline assessment was conducted from March to August 2016; all start time and scheduled time of the first surgeries of each day within that period were collected. The time of arrival of nurses, surgeons and anesthetists were also recorded. An intervention was implemented from September to November 2016. Post-intervention data was collected between December 2016 and March 2017.

\section{Sample}

All first elective surgeries of the day during the preand post-intervention data collection periods were included in our sample. Based on the monthly census, we estimated about 96 first elective surgeries would be included in each of the pre- and post-intervention samples. The scheduled time and surgery start time (first-cut) were extracted from the OR registration book. Emergency cases were excluded from the study as they were not scheduled.

\section{Intervention}

Our quality improvement project implemented a series of interventions to address the first case start on time. The intervention included three components. 1) Developing a policy on staff arrival time. The policy was developed in collaboration with the OR management team and the hospital human resources department in September 2016. 2) Providing training sessions on the policy. After the policy was approved by the hospital and OR department, training sessions on the new policy was set up to orient the staff. Multiple sessions were held to ensure all staff working at the OR was captured despite working in different shifts. The trainings were also repeated over a few weeks' time to ensure staffs who were on leave could attend at least one training session. The training was a one-hour session and it involved the details of the new policy, the potential disciplinary actions and the supervision mechanism to reinforce the policy. 3) Providing continuous supervision to enforce the policy. After the policy was developed and communicated to all OR staff, OR managers started conducting regular morning rounds to ensure staff were arriving on time.

\section{Ethical considerations}

The project was approved by the Institutional Review Board (IRB) of College of Medicine and Health Science (180/UR-CMHS-SHS/16) and IRB of CHUK (EC/ CHUK/121/2016), as well as the hospital administration and the OR department management team.

\section{Measures}

Three indicators were collected to measure the effect of the intervention.

1) The percentage of first surgeries started on time. The start time was defined as the anesthesia induction time in this project. The surgery was considered started on time when the anesthesia induction time was conducted on or before the scheduled time. 
2) The average duration of delay (Difference between anesthesia induction time and scheduled time in minutes).

3) The percentage of staff arrived on time (subdivided by nurses, surgeons and anesthetists). On-time arrival was defined as when the staff arrival time was on or before the time they are required to arrive at the $\mathrm{OR}$.

\section{Data analysis}

Descriptive statistics were used to summarize the findings. Chi-square test was used to compare the preand post-intervention percentage of first surgery on time and percentage of staff arrived on time, and T-tests were used to compare the pre- and post-intervention average duration of surgery delay. All tests were conducted using SPSS v.21 software, with $\mathrm{P}$-value set at 0.05 .

\section{Results}

The start time of 96 first surgeries were collected in the six ORs in the pre-intervention period and 96 in the post-intervention period. The intervention significantly increased the percentage of first surgeries started on time from $3(3 \%)$ pre-intervention to $26(25 \%)$ postintervention, with $\mathrm{P}<0.001$ (Table 1). The average duration of delay was also significantly reduced from 86 minutes pre-intervention to 31 minutes post-intervention, with $\mathrm{P}<0.001$ (Table 1). The percentage of anesthetists who arrived at OR on time increased from $16 \%$ preintervention to $51 \%$ post-intervention $(\mathrm{P}<0.001)$. The percentage of nurses who arrived at OR on time increased from $10 \%$ pre-intervention to $88 \%$ post-intervention $(\mathrm{P}<0.001)$. The percentage of surgeons arrived at OR on time increased from $30 \%$ pre-intervention to $57 \%$ postintervention $(\mathrm{P}<0.001)$ (Table 1).

Table1. Table summarizing the pre- and post-intervention results

\begin{tabular}{|c|c|c|c|c|c|c|}
\hline \multicolumn{3}{|l|}{ Indicators } & Pre intervention & Post intervention & Change & $P$ value \\
\hline \multicolumn{3}{|l|}{ Sample size $(\mathrm{N})$} & 96 & 96 & - & - \\
\hline \multirow{2}{*}{\multicolumn{2}{|c|}{ Surgeries started on time $\mathrm{n}(\%)$}} & Yes & $3(3 \%)$ & $26(27 \%)$ & $24 \%$ & $<0.001$ \\
\hline & & No & $93(97 \%)$ & $70(73 \%)$ & & \\
\hline \multicolumn{3}{|c|}{ Average duration of delay (Minutes) } & 86 & 31 & 55 & $<0.001$ \\
\hline \multirow{6}{*}{$\begin{array}{l}\text { Staff who arrived on } \\
\text { time }\end{array}$} & \multirow[t]{2}{*}{ Anaesthetists } & Yes & $16(17 \%)$ & $49(51 \%)$ & $35 \%$ & $<0.001$ \\
\hline & & No & $80(83 \%)$ & $47(49 \%)$ & & \\
\hline & \multirow[t]{2}{*}{ Nurse } & Yes & $10(10 \%)$ & $84(88 \%)$ & $78 \%$ & $<0.001$ \\
\hline & & No & $86(90 \%)$ & $12(13 \%)$ & & \\
\hline & \multirow[t]{2}{*}{ Surgeon } & Yes & $29(30 \%)$ & $55(57 \%)$ & $27 \%$ & $<0.001$ \\
\hline & & No & $67(70 \%)$ & $41(43 \%)$ & & \\
\hline
\end{tabular}

\section{Discussion}

Healthcare costs are escalating worldwide.[2,20] Quality improvement projects can help reductions in costs in health care delivery while increasing quality.[9] This quality improvement project was designed to reduce delays in starting time in OR at CHUK referral hospital, Kigali. By following the SPS approach, a project team was formed to tackle the issue. The team first identified the problem statement, set objective and conducted a root cause analysis. The OR day-to-day operation involves multiple professional groups and complex work processes. When conducting quality improvement projects in OR, the project team could easily be overwhelmed and distracted by the numerous and convoluted existing problems exist in the OR. By clearly defining the problem statement, the project team was able to focus to address a single issue rather than being distracted by the overwhelming number of problems that OR was facing.

Setting clear objectives helped the team to focus their effort in the right direction, while using available resources to realistically address the issue. In a complex health care setting, naturally there are many root causes contributing to the issue of delay. By conducting the root-cause analysis, the team was able to identify one of the major root causes contributing to the delay in first surgery was due to the late arrival of OR staff. With this root cause identified, creation of appropriate intervention was possible. After analyzing and comparing various potential options, the team decided to implement an intervention that was feasible to execute and designed specifically to address the root cause-develop and enforce a policy to ensure OR staff arrive at work on time to prepare for the first surgeries in order to ensure first surgeries started on time. In public hospitals of Rwanda, morning shift starts at 7:00AM. While many staff arrived at the hospital at 7:00AM, they did not arrive at the work location and start working at the expected time. Without a written policy, there was no enforceable standard.

While the policy was specifically developed based on the root cause in this project, the intervention did not stop at just creating the policy, but included providing training sessions to the OR staff to ensure they understand the policy, and supportive supervision to ensure the policy was operationalized. After the policy was developed, it 
was circulated in the OR for feedback and appropriate modifications were made. Support from the senior management team of the hospital was also essential. The project team and the OR department managers explained to the hospital management team about the nature and objective of the project and the hospital management team was supportive of the effort. By combining the bottom-up (buy-in from staff) and topdown approach (sign off by the management team), the implementation was met with a lot less resistance than anticipated. The intervention successfully increased the percentage of surgeries starting on time with the average duration in delay shortened. The percentages of staff arriving at work on time were also significantly improved across all three disciplines.

Although our study using SPS approach was found successfully in improving the percentage of surgery started on time, there are many other studies which successfully produced similar results using different approaches. For example, a study conducted in Harrisburg hospitals in USA successfully improved the starting time in OR from $13 \%$ to $80 \%$ in one hospital and from $7 \%$ to $83 \%$ in another hospital by applying the six sigma approach.[11] Although the approaches may be different, all these projects emphasized on using data as evidence to inform decision making, indicating the importance of accurate data in improving quality of services at hospitals.

This project addressed one root cause of the delay in surgery start time, however, we did not meet the hospital standard of $90 \%$.[5] This project only addresses the issue about staff arrival time but there were other factors contributing to the delay in starting time of the OR that were found during the study, including breakdown of equipment, lack of communication between operating team members, and long set up time in preparing the operating room. We cannot definitively conclude the improvement was due to our quality improvement effort as we cannot control confounding factors. More quality improvement projects using SPS should be conducted to address the other root causes in order to further enhance the timeliness of starting first surgeries and thus improve the efficiency of the OR and quality of care. The study was only conducted in the OR department in one hospital, the results may not be generalizable to other settings.

\section{Conclusion}

Following the eight-step Strategic Problem Solving approach can help identify the factors influencing first case delays and thus create appropriate interventions to address the problem. More quality improvement projects should be conducted in health care settings using similar approach to address other root causes as well as other problems. Although the project showed positive results, long term sustainability was not assessed; longer term evaluation should be conducted to evaluate the sustainability.

\section{Acknowledgements}

We thank the OR team at CHUK in allowing us to conduct the project in their department and providing us the needed data.

\section{Conflicts of interest}

No conflict of interest.

This article is published open access under the Creative Commons Attribution-NonCommercial NoDerivatives (CC BY-NC-ND4.0). People can copy and redistribute the article only for noncommercial purposes and as long as they give appropriate credit to the authors. They cannot distribute any modified material obtained by remixing, transforming or building upon this article. See https://creativecommons.org/ licenses/by-nc-nd/4.0/

\section{References}

1. Schuster M, Pezzella M, Taube C, Bialas E, Diemer M, Bauer M. Delays in Starting Morning Operating Lists. 2013;110:237-43.

2. Does RJMM, Vermaat TMB, Verver JPS, Bisgaard $\mathrm{S}$, Heuvel JVD. Reducing Start Time Delays in Operating Rooms. J Qual Technol [Internet]. 2009;41:95-109. Available from: http://dare.uva.nl/ document/2/62327

3. Wright JG, Roche A, Ann Roche RN, Khoury AE, Roche A. Improving on-time surgical starts in an operating room. Can J Surg. 2010;53:167-70.

4. Varughese AAM, Adler E. Improving On-Time Start of Day and End of Day for a Pediatric Surgical Service. 2013;132.

5. Gupta B, Nita D, Soni KD, Pramendra Agrawal, Nita D'souza KDSD. Start time delays in operating room: Different perspectives. Saudi J Anaesth [Internet]. 2011;5:286. Available from: http://www.saudija. org/text.asp?2011/5/3/286/84103

6. Mehta P, Mahmoud A, El-sergany A, Culberson D. Improving operating room start times in a community teaching hospital. 2016;5:1-7.

7. Roberts S, Saithna A, Bethune R. Improving theatre efficiency and utilisation through early identification of trauma patients and enhanced communication between teams. BMJ Qual Improv Reports. 2015;4:u206641.w2670-u206641.w2670.

8. Thayer C, Thayer C. Surgical services optimization: First case starts and turnover time. 2012;

9. Garner P. Complexities in the Operating Room. Proc 2012 Ind Syst Eng Res Conf. 2012; 
10. Pandit JJ, Westbury S, Pandit M. The concept of surgical operating list 'efficiency': A formula to describe the term. Anaesthesia. 2007;62:895-903.

11. Parvathaneni A, O. Peter A, Wilson C. Wheels on Time: A Six Sigma Approach to Reduce Delay in Operating Room Starting Time. Surg Curr Res [Internet]. 2011;01:1-4. Available from: http:// www.omicsonline.org/2161-1076/2161-1076-1102.digital/2161-1076-1-102.html

12. Wong J, Khu KJ, Kaderali Z, Bernstein M. Delays in the operating room: Signs of an imperfect system. Can J Surg. 2010;53:189-95.

13. Ibyimana C, Wong R, Adomako E, Lukas S, Birungi F, Munyanshongore C. Establishing a tracking system in human resources department to improve the completeness of personnel files at a district hospital in Rwanda. 2016;5.

14. Nyirasafari R, Corden MH, Karambizi AC, Kabayiza C, Makuza JD, Wong R, et al. Paediatrics and International Child Health care unit in Kigali , Rwanda Predictors of mortality in a paediatric intensive care unit in Kigali, Rwanda. Paediatr Int Child Health [Internet]. Taylor \& Francis; 2016;9047:1-7. Available from: http://dx.doi.org/1

\section{$0.1080 / 20469047.2016 .1250031$}

15. Nsengiyumva JP, Wong R, Adomako E, Pawelzik V, Yerger J, Uwitonze E, et al. ORIGINAL ARTICLE A quality improvement project to reduce intravenous catheter related infections in the neonatology unit of Kibogora hospital in Rwanda. 2016;5.

16. Bahufite A, Ndagijimana A, Adomako E, Zerihun A, Simba CA, Ntakirutimana C, et al. Implementing wound dressing protocol to reduce post cesarean section surgical site infections in Mibilizi District Hospital, Rwanda. Horiz. 2016;24:369-76.

17. Banaszak-Holl J, Nembhard IM, Taylor L BE. "Leadership and management: a framework for action", Shortell and Kaluzny's Healthcare Management: Organizational Theory and Behavior. 2011.

18. Ministry of Health R of R. Annual report July 2012June 2013. 2013.

19. UTHK/CHUK. Health Management Information System(HMIS) Surgery department. 2015.

20. Mclaughlin MM. a Model To Evaluate Efficiency in Operating Room Processes. 2012;200. 